\title{
Worse Outcomes among African Americans with Liver Cancer: What Might Cause the Disparity?
}

\author{
Winters AC ${ }^{1}$, Perumalswami PV ${ }^{2}$ and Branch $\mathrm{AD}^{2 *}$ \\ ${ }^{1}$ Department of Medicine, Icahn School of Medicine at the \\ Mount Sinai Medical Center, USA \\ ${ }^{2}$ Division of Liver Disease, Icahn School of Medicine at \\ the Mount Sinai Medical Center, USA \\ *Corresponding author: Branch $\mathrm{AD}$, Division of Liver \\ Disease, Icahn School of Medicine at the Mount Sinai \\ Medical Center, 1425 Madison Avenue, $11^{\text {th }}$ Floor, Room \\ 24, New York, NY 10029, USA
}

Received: February 25, 2017; Accepted: March 30, 2017; Published: April 10, 2017

\begin{abstract}
Liver cancer remains an important cause of cancer mortality in the United States. Incidence of hepatocellular carcinoma (HCC), the most common histological cell type of liver cancer, has increased over the past 30 years. The statistics are particularly worrisome for African Americans. Their incidence of HCC far surpasses that of other races, particularly whites. Additionally, mortality for African Americans with $\mathrm{HCC}$ is also far worse. In a review of the literature, we found a stepwise progression of variables that may contribute to this observation. African Americans have the highest observed rates of infection with chronic hepatitis and are offered treatment less often for hepatitis $C$ virus. The progression to cirrhosis is slower, which may complicate HCC surveillance. Regardless, they receive surveillance either biochemical or radiographic at a lower rate than patients of other races. Further, African Americans present at a more advanced stage and are referred for curative therapy less often. When African Americans do receive therapy, their outcomes are worse. Additional investigation is warranted as to whether differences in tumor biology may also play a role.
\end{abstract}

Keywords: Liver cancer; Hepatocellular carcinoma; Hepatitis C Virus

\section{Introduction}

Liver cancer is the fifth most common cause of cancer in men worldwide and the second leading cause of site-specific cancerrelated death [1]. Hepatocellular carcinoma (HCC) is by far the most common histological cell type of liver cancer, accounting for $90 \%$ of all liver cancer in the United States (US) [2]. According to the NIH Surveillance, Epidemiology, and End Results (SEER) database, the annual age-adjusted incidence of HCC in the US is increasing rapidly. It more than tripled between 1975 and 2005 rising from 1.6 per 100,000 to 4.9 per 100,000 [2]. The statistics are particularly worrisome for African Americans. According to the SEER database, their HCC incidence in 2005-2007 was 7.6 per 100,000 over 1.5 -fold higher than the national average [3]. Key risk factors for HCC include liver cirrhosis (typically due to chronic liver disease) and male sex rates of liver cancer in men are two to four times as high as those in women [4]. HCC arises in a background of liver cirrhosis in 80$90 \%$ of cases, [5] with the strength of association dependent on the etiology of the liver disease [6].

As of 2008, the worldwide incidence and mortality of liver cancer were essentially equal, highlighting the urgent need for better strategies for prevention, early diagnosis, and treatment. The prognosis for most patients with HCC is grim 5 year survival ranges between 6-7\% [7] worldwide and is less than $12 \%$ in the US [3]. Early detection allows life-saving interventions to be offered. With curative surgery, HCCs detected at very early stages have 5-year survival rates reported over 50\% [8]. In an effort to increase the likelihood that HCC will be detected at an early and curable stage, guidelines of the American Association for the Study of Liver Disease (AASLD) recommend life-long bi-annual screening with ultrasound for patients with liver cirrhosis [9].
There are a number of worrisome trends for African Americans with respect to HCC. Black patients with cirrhosis receive less surveillance for HCC than other racial groups particularly nonHispanic Caucasians [10]. Perhaps as a consequence, African Americans have more advanced tumor stage at diagnosis and receive definitive surgical treatment less often than other racial groups. Most concerning is that African Americans with HCC have higher mortality than other ethnicities [11-16]. This review examines the factors that might contribute to increased HCC risk and mortality among African Americans. These include greater exposure to agents that cause liver damage (e.g. chronic hepatitis $\mathrm{C}$ virus (HCV) infection and hepatitis B virus (HBV) infection), less access to healthcare [14] and, potentially, biological and histological differences in the HCC tumor subtype.

\section{Potential contributors to the elevated HCC risk and higher mortality in african americans}

Higher exposure to factors causing chronic liver injury:

HCV infection: The relationship between $\mathrm{HCV}$ infection and HCC risk in African Americans is complex and incompletely understood. African Americans have a higher prevalence of chronic HCV infection [17] than that of any other racial group in the US. This is in part due to a polymorphism in the interferon (IFN) lambda 4 gene that reduces rates of spontaneous clearance and reduces the effectiveness of interferon-based treatments, which were the mainstay of HCV treatment until about two years ago [18]. The lower response rate to IFN-based treatment may have discouraged HCV-treatment during the many years when they were the only therapies available.

The National Health and Nutrition Examination Survey (NHANES) III, which examined HCV rates in the US from 19881994, found that non-Hispanic blacks had the highest prevalence of 
HCV exposure: $3.2 \%$ with HCV antibodies compared to $1.5 \%$ among non-Hispanic whites [19]. In a subsequent NHANES survey, from 1999-2002, the prevalence amongst blacks remained twice the rate of non-Hispanic whites (3.0\% vs. 1.5\%) [19]. This survey also found that the highest prevalence of HCV was in non-Hispanic black men between the ages of $40-49$ years $13.6 \%$ versus roughly $6 \%$ for nonHispanic white men and around $10 \%$ for Mexican-American men within the same age group $[19,20]$.

Among 149,407 patients with confirmed HCV infection defined as a positive HCV-antibody and an HCV associated ICD-9 code between 1999-2010 in the Veterans Administration (VA) HCV Clinical Case Registry, $36.1 \%$ were African American, second only to non-Hispanic whites, who comprised $56.3 \%$ of those in the registry [21].

Interestingly, within this VA cohort, El-Serag, et al. found that HCV-positive African Americans had a lower risk of both progression to cirrhosis [Hazards Ratio (HR): $0.58,95 \%$ Confident Interval (CI) $=0.55-0.61]$ and development of HCC [HR: 0.77, 95\% CI=0.71-0.83] than HCV-positive Caucasians. These data were adjusted for year of HCV diagnosis, age, gender, HCV genotype, body mass index, alcohol use, HIV, HBV coinfection, period of service, and HCV treatment response [21]. Other studies also demonstrated a lower risk of progression to cirrhosis in African Americans, though they have failed to reach statistical significance [17]. These findings could be problematic if HCC risk in African Americans is not as closely tied to liver cirrhosis as it is in other racial/ethnic groups. As mentioned, HCC screening guidelines target patients with cirrhosis because the vast majority of cancer arises in the cirrhotic liver ( 80-90\%) [4]. These findings could have a deleterious effect if it results in failure to be engaged in HCC screening and, thus, delaying diagnoses.

In addition to increased incidence, African Americans are treated less for chronic HCV. El-Serag, et al. found that African Americans were the least likely to have undergone antiviral therapy with ribavirin and IFN, with almost $90 \%$ of their population never receiving treatment compared to $81 \%$ for non-Hispanic whites [21]. Though, again, under the old paradigm of treatment, African American patients had lower rates of sustained virological response (SVR), perhaps influencing provider decision-making and thus contributing to these data.

DAAs for HCV bring hope to millions around the globe with $\mathrm{HCV}$ with their excellent efficacy. There is evidence, however, that African American patients receive DAAs less often than white patients. In one study of the veteran population with HCV seeking care in the VA system during the DAA era, Kanwal, et al. found that black patients had 22\% lower odds [Odds Radio (OR): 0.78; 95\% CI: $0.73-0.84$ ] of being treated with DAAs than white patients [22]. This difference persisted after adjustment for socioeconomic status, severity of liver disease, comorbidity and healthcare utilization. Furthermore, this disparity persisted even in patients presenting to $\mathrm{HCV}$-specialty clinics. Black patients in this study had higher rates of HIV, homelessness, diabetes and drug and alcohol use, which are all predictors of DAA treatment.

When African Americans are successfully treated, they may maintain an elevated risk of developing HCC. In a study of veterans who received IFN-based treatment for HCV and achieved a sustained virological response (SVR), indicative of HCV cure, El-Serag, et al. found that black patients had an increased risk of developing HCC compared to whites HR of 1.4 but the increase was not statistically significant [ $p=0.31$ ] [23]. Many of the studies on post-SVR HCC were done in Japan $[24,25]$ and data are limited on risk factors for postSVR HCC in direct acting antiviral (DAA) users.

\section{Increased prevalence of $\mathrm{HBV}$ infection in african americans}

HBV is an important cause of HCC even in the absence of cirrhosis. The exact mechanism linking chronic HBV infection with HCC is not fully understood. Progression to cirrhosis remains an important risk factor however, in the US, one study estimated that the annual incidence of HCC in non-cirrhotic patients with chronic HBV infection is $0.3 \%$, [26] indicating that other factors play a role. HBV DNA becomes integrated into the chromosomes of infected hepatocytes including locations associated with cellular growth [27]. Additionally, the HBV viral X protein has been implicated in the dysregulation of cellular growth control mechanisms [27].

African Americans have the highest incidence of acute HBV of any group in the United States 1.7 cases per 100,000 persons as of 2010 [20]. The majority of cases of acute HBV results from horizontal transmission and the vast majority (95\%) are cleared and do not result in chronic HBV infection [28]. According to data in the NHANES III (1988-1994) and a subsequent NHANES (1996-2006) survey, ageadjusted prevalence of chronic HBV infection defined as patients with positive anti-HBc and HBsAg in the non-Hispanic black population increased from $0.83 \%$ to $0.89 \%$, the only group studied that showed an increase over the time surveyed [29]. For comparison, during the same period rates of chronic HBV in non-Hispanic whites fell from $0.21 \%$ to $0.09 \%$ [29].

\section{Alcohol and diabetes potentiate HCC risk}

Heavy alcohol consumption is an independent risk factor for HCC [30]. Additionally, alcohol has immunologic and oxidativestress related consequences on the livers of patients who are infected with $\mathrm{HCV}$ [31]. It's controversial whether diabetes mellitus (DM) increases HCC risk in the absence of other causes of liver disease $[30,32,33]$. There is, however, strong evidence that DM potentiates HCC risk in patients with underlying chronic liver disease [32,34].

In a case-control study of 115 patients with HCC and 230 without, Hassan et al. found a significant, independent association between the presence of DM both non-insulin and insulin dependent and the risk of HCC [adjusted OR 4.3, 95\% CI=1.9-9.9] [30]. Within the same population, a history of heavy alcohol consumption (defined as $>80 \mathrm{ml}$ ethanol/day) was an independent risk factor for HCC [adjusted OR 2.4, 95\% CI=1.3-4.4]. When a diagnosis of DM was combined with the presence of viral hepatitis, a statistically significant synergistic relationship was found on the incidence of HCC. A similar relationship was found between heavy alcohol consumption with the presence of DM and incidence of HCC [30]. In a population of military veterans, El-Serag, et al. showed similar synergism between the presence of DM and either HBV, HCV or alcoholic cirrhosis and the incidence of HCC [OR 1.57, p=0.018] [32]. This study did not find an independent association between DM and HCC, however. There is recent evidence that the relationship between liver disease and DM in cirrhosis may only apply to cirrhotic patients with non-HCV liver 
disease, but more studies are needed [34].

This synergism has important implications for the African American population. In a veteran population infected with $\mathrm{HCV}$, African Americans had a significantly higher prevalence of alcohol abuse (59.2\% vs. $49.5 \%)$ and DM (16.1\% vs. 8.7\%) than non-Hispanic whites. This difference in potentiating factors predisposes the African American population to greater HCC risk and may partially explain the increased prevalence observed.

Lower HCC surveillance rates in african americans: The AASLD recommends life-long biannual ultrasound surveillance in cirrhotic patients and some non-cirrhotic HBV-positive patients [9]. Some authors and societies advocate $[4,35]$ the measurement of serum alpha fetoprotein (AFP) levels in conjunction with ultrasound surveillance; however, when the most-commonly used cut-point, $20 \mathrm{ng} / \mathrm{mL}$, is applied, AFP has less sensitivity than is optimal for a screening modality 25-65\% [4] explaining the reluctance to include AFP testing in routine HCC screening [36,37]. Despite the limitations, the National Comprehensive Cancer Network recommends all cirrhotic patients and $\mathrm{HBV}$-carriers undergo both US and serum alpha-fetoprotein (AFP) testing every 6-12 months [35]. The American College of Gastroenterology also recommends ultrasound and AFP testing, though at unstated intervals [35]. In addition to sub-optimal sensitivity, AFP measurements also have sub-optimal specificity. Sterling, et al. found that in a group of 855 patients with advanced $\mathrm{HCV}$, about $25 \%$ of patients without HCC had an AFP $>20 \mathrm{ng} / \mathrm{mL}$ [38].

An AFP cut-off of $20 \mathrm{ng} / \mathrm{mL}$ has an especially low sensitivity among African Americans with chronic HCV infection. In one retrospective case-control study of HCV patients with HCC, Nguyen, et al. found that African American patients with HCV and HCC were only 56\% more likely to a "more abnormal" AFP level than African American patients with HCV alone [39]. The authors concluded that "AFP [was] of no value in this group of patients" [39]. This group also found that at an AFP cutoff of $10 \mathrm{ng} / \mathrm{mL}$, the sensitivity for detecting HCC was $57.1 \%$ for African Americans and $81.6 \%$ for non-African Americans ( $\mathrm{p}$-value $=0.02$ ). At a cutoff of $20 \mathrm{ng} / \mathrm{mL}$, the sensitivities for the two groups were $42.9 \%$ and $66 \%$ respectively (p-value $=0.05$ ). It is possible that AFP measurement will be a more sensitive and specific indicator of HCC in patients who have been cured of HCV than among patients with active, chronic $\mathrm{HCV}$ infection as HCV cure is accompanied by a decrease in AFP levels [40].

Troublingly, African Americans at risk for HCC are screened less than other groups. Among a population of roughly 1,800 cirrhotic patients in the SEER database with an eventual diagnosis of HCC between 1994 and 2002, Davila, et al. found that a smaller percentage of black patients received any screening as compared to white patients (40.8\% versus 53.3\%) [10]. "Any screening" in this case was defined as ever having an AFP level measured or an abdominal ultrasound. Black patients also had lower rates of "regular surveillance" defined as an annual surveillance test, ultrasound and/or AFP at least 2 of the 3 years prior to HCC diagnosis and "inconsistent surveillance" defined as one surveillance test 2-3 years prior to diagnosis. The authors were unable to examine data on tests ordered and not completed, thus it is unclear how much adherence plays a role in the observed screening rates.
Similarly, among patients in the national VA Hepatitis C Clinical Case Registry, black patients were less likely than any other racial group to undergo routine surveillance (OR 0.6, 95\% CI=0.45-0.81) defined as either AFP or ultrasound in two consecutive years within the first four years after diagnosis of cirrhosis [41].

\section{Differences in presentation, treatment and survival among african americans}

Black patients present with HCC at a younger median age and with more advanced tumor stages than white patients. In a review of HCC in white and black patients, Sloane, et al. found a greater proportion of black patients presented prior to age 70 years than white patients $(\mathrm{p}<0.0001)[11]$.

Treatment options for HCC depend on the size, location and spread of the cancer as well as the severity of the underlying liver disease. Partial hepatectomy is potentially curable and is optimal for patients without distant metastases and adequate liver function. Patients with low hepatic reserve who cannot tolerate hepatectomy may undergo orthotopic liver transplantation under certain circumstances. Selection for transplant is based upon tumor criteria such as the Milan criteria, which includes patients who have a solitary tumor $<5 \mathrm{~cm}$, or 3 lesions $<3 \mathrm{~cm}$ and localized disease without vascular invasion [42]. Other treatments for disease that remains within the liver include radiofrequency ablation or transarterial chemoembolization, often used alone or as a bridge to transplant. Systemic chemotherapy is an option for patients with HCC that has spread outside the liver; however the most successful agent, sorafenib, extends survival for only about 3 months, from a median of 7.9 months to 10.7 months [43].

Data suggest African Americans present with more advanced tumor stages, limiting treatment options. In the Sloane, et al. study mentioned above, black patients were more likely to have regional (27.5\% versus $22.4 \%)$ and distant (22.7\% versus $19.9 \%)$ metastases $(\mathrm{p}<0.0005)$ at diagnosis.

Multiple studies have shown that black patients are less likely to receive surgical management of HCC, which is generally an option for patients with better preserved liver function [11,14-16]. Sloane and his colleagues reported that black patients with localized HCC defined as disease confined to the initial organ without evidence of spread were more likely to have "surgery not recommended" (45\% versus about $37 \%)$ and less likely to have "surgery performed" (20\% versus $30 \%)$ when compared to white patients $(\mathrm{p}<0.001)$ [11]. In patients with regional spread nearby lymph nodes, organs or tissues black patients had surgery "not recommended" $60 \%$ of the time compared to $55 \%$ in whites $(\mathrm{p}=0.012)$. The investigators proposed advanced stage as presentation may have contributed to this disparity, but stage alone cannot account for the differences in patients with localized disease.

Mathur, et al. found that black and Hispanic patients received invasive therapy defined as tumor ablation, hepatectomy or liver transplant at lower rates than whites and Asians $(\mathrm{p}<0.001)$ [14]. The authors cited poor access to complex cancer care as a potential contributor to this disparity. Hoehn, et al. however, found that black patients with localized HCC were less likely to receive surgery even after adjusting for income, education, insurance status and stage at presentation [16]. Presence of comorbidities that may increase 


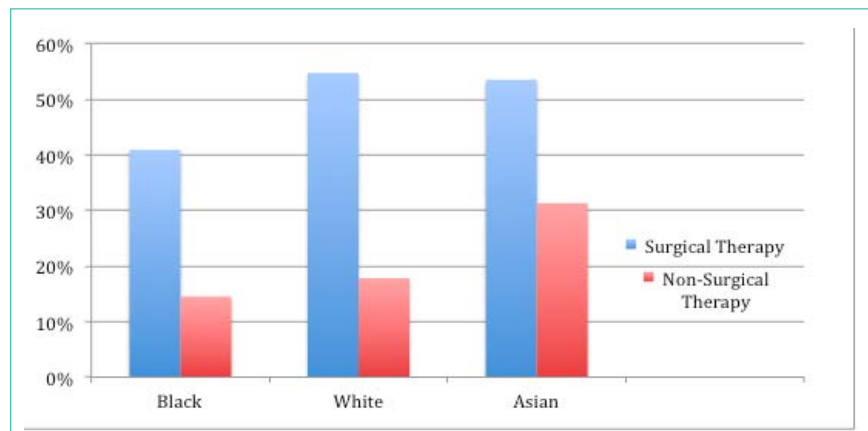

Figure 1: 5-year survival after surgical therapy (resection/transplant, blue) and non-surgical therapy (ablation, red) is lower in Blacks compared to White and Asians [15].

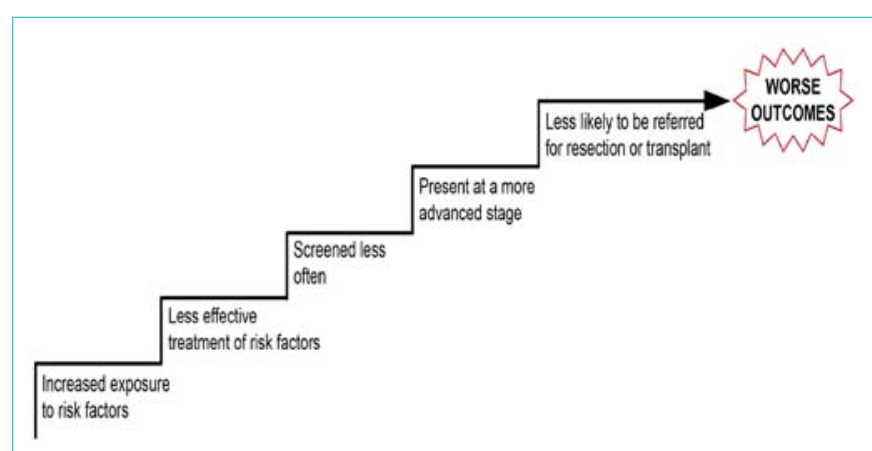

Figure 2: Stepwise progression towards worse outcomes for African Americans with HCC.

surgical risks was offered as one possible explanation, though the authors were unable to find data to support this inference.

It is also well established that black patients with HCC have lower survival rates $[11,14-16]$. Sloane, et al. found that among their cohort, survival for black patients with HCC was lower than for white patients after adjusting for age, gender, tumor stage and surgical treatment intervention used ( $\mathrm{HR}$ 95\% CI=1.041-1.2, $\mathrm{p}=0.002$ ). Similarly, when compared to Asian and white patients, Hoehn, et al. found that black patients have higher mortality after adjusting for stage and socioeconomic factors [16]. Xu, et al. showed black patients who underwent surgery had lower 5-year survival than white and Asian and patients (Figure 1) [15]. Survival was also worse for black patients undergoing non-surgical therapy (Figure 1). Mathur, et al. found that black race was a significant predictor of poor outcome from invasive therapy defined as tumor ablation, hepatic resection or transplant, which maintained statistical significance even after adjusting for tumor stage, socioeconomic and clinical status [14]. There was no difference in mortality amongst patients who received a liver transplant, though they noted that black patients were two-fold less likely to receive liver transplants than white patients.

Finally, in a recent comprehensive look at inpatient hospitalizations in 2011 for HCC, Rajbhandari, et al. showed an alarming disparity in inpatient mortality for black patients [44]. Overall, $10.9 \%$ of inpatient admissions for black patients ended in death, compared to $6.4 \%$ for whites (OR 1.8, 95\% CI=1.3-2.47, $\mathrm{p}<0.001)$. This difference remained after adjustment for the presence of metastatic disease, receipt of tumor-related procedures and both systemic and liver-related inpatient complications. With regard to socioeconomic considerations, the difference persisted despite adjustment for insurance status and zip code income level of the patients studied.

\section{Conclusion}

The incidence of HCC among African Americans in the US is increasing. Their exposure to the number one risk factor in the US $\mathrm{HCV}$ is the highest of any racial group. When African Americans are diagnosed with HCC, their tumors are diagnosed at a later stage, and are more widespread. African Americans receive potentially curative surgery less often and their outcomes are worse than non-African Americans. The reasons for these disparities are not entirely clear, though socioeconomic factors and access to care appear to contribute. Figure 2 depicts a confluence of these factors that contribute to the observed disparity.

Further research is needed to determine whether distinctive tumor biology also plays a role. A biological component is suggested by the younger age at onset of HCC, differences in AFP levels and differing response to therapy in African Americans. Additionally, data on differences in HCC tumor histology among racial groups is sparse, though other diseases may set some precedent. African American women who develop breast cancer, for example, have a higher incidence of triple-negative breast cancer than other racial groups [45]. Expounding upon differences in liver tumor biology and histology amongst racial groups in a similar fashion may help explain some of these disparities as well as how social determinants of healthcare impact biology.

\section{References}

1. Torre LA, Bray F, Siegel RL, Ferlay J, Lortet-Tieulent J, Jemal A. Global cancer statistics, 2012. CA Cancer J Clin. 2015; 65: 87-108.

2. Altekruse SF, McGlynn KA, Reichman ME. Hepatocellular carcinoma incidence, mortality, and survival trends in the United States from 1975 to 2005. J Clin Oncol. 2009; 27: 1485-1491.

3. Mittal S, El-Serag HB. Epidemiology of hepatocellular carcinoma: consider the population. J Clin Gastroenterol. 2013; 47: S2-S6.

4. El-Serag HB. Hepatocellular carcinoma. N Engl J Med. 2011; 365: 11181127.

5. El-Serag HB. Epidemiology of viral hepatitis and hepatocellular carcinoma. Gastroenterology. 2012; 142: 1264-1273.

6. White DL, Kanwal F, El-Serag HB. Association between nonalcoholic fatty liver disease and risk for hepatocellular cancer, based on systematic review. Clin Gastroenterol Hepatol. 2012; 10: 1342-1359.

7. Artinyan A, Mailey B, Sanchez-Luege N, Khalili J, Sun CL, Bhatia S, et al. Race, ethnicity, and socioeconomic status influence the survival of patients with hepatocellular carcinoma in the United States. Cancer. 2010; 116: 13671377.

8. Song T. Recent advances in surgical treatment of hepatocellular carcinoma. Drug Discov Ther. 2015; 9: 319-330.

9. Bruix J, Sherman M. Practice Guidelines Committee, American Association for the Study of Liver Diseases. Management of hepatocellular carcinoma. Hepatology. 2005; 42: 1208-1236.

10. Davila JA, Morgan RO, Richardson PA, Du XL, McGlynn KA, El-Serag $\mathrm{HB}$. Use of surveillance for hepatocellular carcinoma among patients with cirrhosis in the United States. Hepatology. 2010; 52: 132-141.

11. Sloane D, Chen H, Howell C. Racial disparity in primary hepatocellular carcinoma: tumor stage at presentation, surgical treatment and survival. J Natl Med Assoc. 2006; 98: 1934-1939. 
12. Njei B, Rotman Y, Ditah I, Lim JK. Emerging trends in hepatocellular carcinoma incidence and mortality. Hepatology. 2015; 61: 191-199.

13. Wong RJ, Corley DA. Survival differences by race/ethnicity and treatment for localized hepatocellular carcinoma within the United States. Dig Dis Sci. 2009; 54: 2031-2039.

14. Mathur AK, Osborne NH, Lynch RJ, Ghaferi AA, Dimick JB, Sonnenday CJ. Racial/ethnic disparities in access to care and survival for patients with earlystage hepatocellular carcinoma. Arch Surg. 2010; 145: 1158-1163.

15. Xu L, Kim Y, Spolverato G, Gani F, Pawlik TM. Racial disparities in treatment and survival of patients with hepatocellular carcinoma in the United States. Hepatobiliary Surg Nutr. 2016; 5: 43-52.

16. Hoehn RS, Hanseman DJ, Wima K, Ertel AE, Paquette IM, Abbott DE, et al Does race affect management and survival in hepatocellular carcinoma in the United States? Surgery. 2015; 158: 1244-1251.

17. Pearlman BL. Hepatitis C virus infection in African Americans. Clin Infect Dis. 2006; 42: 82-91.

18. O'Brien TR, Prokunina-Olsson L, Donnelly RP. IFN-|̂̀»4: the paradoxical new member of the interferon lambda family. J Interferon Cytokine Res. 2014; 34: 829-838.

19. Armstrong GL, Wasley A, Simard EP, McQuillan GM, Kuhnert WL, Alte MJ. The prevalence of hepatitis C virus infection in the United States, 1999 through 2002. Ann Intern Med. 2006; 144: 705-714.

20. Forde KA, Tanapanpanit O, Reddy KR. Hepatitis B and C in African Americans: current status and continued challenges. Clin Gastroenterol Hepatol. 2014; 12: 738-748.

21. El-Serag HB, Kramer J, Duan Z, Kanwal F. Racial differences in the progression to cirrhosis and hepatocellular carcinoma in HCV-infected veterans. Am J Gastroenterol. 2014; 109: 1427-1435

22. Kanwal F, Kramer JR, El-Serag HB, Frayne S, Clark J, Cao Y, et al. Race and Gender Differences in the Use of Direct Acting Antiviral Agents for Hepatitis C Virus. Clin Infect Dis. 2016; 63: 291-299.

23. El-Serag HB, Kanwal F, Richardson P, Kramer J. Risk of Hepatocellula Carcinoma after Sustained Virologic Response in Veterans with HCVinfection. Hepatology. 2016; 64: 130-137.

24. Ikeda M, Fujiyama S, Tanaka M, Sata M, Ide T, Yatsuhashi H, et al. Risk factors for development of hepatocellular carcinoma in patients with chronic hepatitis C after sustained response to interferon. J Gastroenterol. 2005; 40 $148-156$

25. Toyoda H, Kumada T, Tada $T$, Kiriyama S, Tanikawa M, Hisanaga $Y$, et al. Risk factors of hepatocellular carcinoma development in non-cirrhotic patients with sustained virologic response for chronic hepatitis C virus infection. Gastroenterol Hepatol. 2015; 30: 1183-1189.

26. Fattovich G, Bortolotti F, Donato F. Natural history of chronic hepatitis B special emphasis on disease progression and prognostic factors. J Hepatol. 2008; 48: 335-352.

27. Di Bisceglie AM. Hepatitis B and hepatocellular carcinoma. Hepatology. 2009; 49: S56-S60.

28. Kumar M, Satapathy S, Monga R, Das K, Hissar S, Pande C, et al. A randomized controlled trial of lamivudine to treat acute hepatitis $B$. Hepatology. 2007; 45: 97-101.
29. Wasley A, Kruszon-Moran D, Kuhnert W, Simard EP, Finelli L, McQuillan G, et al. The prevalence of hepatitis B virus infection in the United States in the era of vaccination. J Infect Dis. 2010; 202: 192-201.

30. Hassan MM, Hwang LY, Hatten CJ, Swaim M, Li D, Abbruzzese JL, et al. Risk factors for hepatocellular carcinoma: synergism of alcohol with viral hepatitis and diabetes mellitus. Hepatology. 2002; 36: 1206-1213.

31. Safdar K, Schiff ER. Alcohol and hepatitis C. Semin Liver Dis. 2004; 24: 305315.

32. El-Serag HB, Richardson PA, Everhart JE. The role of diabetes in hepatocellular carcinoma: a case-control study among United States Veterans. Am J Gastroenterol. 2001; 96: 2462-2467.

33. Wang P, Kang D, Cao W, Wang Y, Liu Z. Diabetes mellitus and risk of hepatocellular carcinoma: a systematic review and meta-analysis. Diabetes Metab Res Rev. 2012; 28: 109-122.

34. Yang JD, Mohamed HA, Cvinar JL, Gores GJ, Roberts LR, Kim WR. Diabetes Mellitus Heightens the Risk of Hepatocellular Carcinoma Except in Patients With Hepatitis C Cirrhosis. Am J Gastroenterol. 2016; 111: 1573-1580.

35. Yu SJ. A concise review of updated guidelines regarding the management of hepatocellular carcinoma around the world: 2010-2016. Clin Mol Hepatol. 2016; 22: 7-17.

36. Sherman M. Serological surveillance for hepatocellular carcinoma: time to quit. J Hepatol. 2010; 52: 614-615.

37. Bruix J, Sherman M. Management of Hepatocellular Carcinoma: An Update. Hepatology. 2011. 53: 1020-1022.

38. Sterling RK, Wright EC, Morgan TR, Seeff LB, Hoefs JC, Di Bisceglie AM, et al. Frequency of elevated hepatocellular carcinoma (HCC) biomarkers in patients with advanced hepatitis C. Am J Gastroenterol. 2012; 107: 64-74.

39. Nguyen MH, Garcia RT, Simpson PW, Wright TL, Keeffe EB. Racial differences in effectiveness of alpha-fetoprotein for diagnosis of hepatocellular carcinoma in hepatitis C virus cirrhosis. Hepatology. 2002; 36: 410-417.

40. Crismale J. Hepatocellular Carcinoma in Patients Cured of Hepatitis C Virus: Less Cirrhosis and Liver Fat than Expected. AASLD Liver Learning. 2016.

41. Singal AG, Yopp A, S Skinner C, Packer M, Lee WM, Tiro JA. Utilization of hepatocellular carcinoma surveillance among American patients: a systematic review. J Gen Intern Med. 2012; 27: 861-867.

42. Mazzaferro V, Bhoori S, Sposito C, Bongini M, Langer M, Miceli R, et al. Milan criteria in liver transplantation for hepatocellular carcinoma: an evidencebased analysis of 15 years of experience. Liver Transpl. 2011; 172: S44-S57.

43. Llovet JM, Ricci S, Mazzaferro V, Hilgard P, Gane E, Blanc JF, et al. Sorafenib in advanced hepatocellular carcinoma. N Engl J Med. 2008; 359: 378-390.

44. Rajbhandari R, Simon RE, Chung RT, Ananthakrishnan AN. Racial Disparities in Inhospital Outcomes for Hepatocellular Carcinoma in the United States. Mayo Clin Proc. 2016; 91: 1173-1182.

45. Dietze EC, Sistrunk C, Miranda-Carboni G, O'Regan R, Seewaldt VL. Triplenegative breast cancer in African-American women: disparities versus biology. Nat Rev Cancer. 2015; 15: 248-254.
Austin J Gastroenterol - Volume 4 Issue 2 - 2017 ISSN : 2381-9219 | www.austinpublishinggroup.com Branch et al. (C) All rights are reserved
Citation: Winters AC, Perumalswami PV and Branch AD. Worse Outcomes among African Americans with Liver Cancer: What Might Cause the Disparity?. Austin J Gastroenterol. 2017; 4(2): 1079. 